\title{
Free Silicone-Induced Deep Inferior Epigastric Perforator Flap Failure in the Setting of Silicone Mastitis
}

\author{
Eric S. Weiss, MD ${ }^{1, *}$ Marilyn Ng, MD ${ }^{2, *} \quad$ Kara E. Friend, MD ${ }^{1} \quad$ Eric I. Chang, MD ${ }^{2} \quad$ Neal S. Topham, MD ${ }^{2}$ \\ Sameer A. Patel, MD² \\ ${ }^{1}$ Department of Surgery, Einstein Healthcare Network, Philadelphia, \\ Pennsylvania \\ ${ }^{2}$ Department of Surgical Oncology, Division of Plastic \& \\ Reconstructive Surgery, Fox Chase Cancer Center, Philadelphia, \\ Pennsylvania \\ Address for correspondence Sameer A. Patel, MD, FACS, Department \\ of Surgical Oncology, Division of Plastic \& Reconstructive Surgery, Fox \\ Chase Cancer Center, 333 Cottman Avenue, Philadelphia, PA 19111 \\ (e-mail: sameer.patel@fccc.edu).
}

J Reconstr Microsurg Open 2017;2:e46-e48.

The illicit use of injectable silicone for cosmetic purposes in the breast has persisted for over five decades without support of the Food and Drug Administration (FDA) ${ }^{1}$ and is associated with local and systemic complications. ${ }^{1-3}$ The constellation of symptoms first described by Boo-Chai in 1969 as "silicone mastitis," 4 stems from an inflammatory reaction to silicone and consists of pain, discoloration, and irregular nodularity. Though there are numerous reports regarding silicone mastitis, ${ }^{4-6}$ the exact incidence is unknown. Unless confined to a localized area of the breast, the most effective treatment is mastectomy. As silicone infiltration increases the risk of tissue ischemia and infection, autologous tissue reconstruction mitigates these risks and theoretically offers a superior option compared with breast implant-based reconstruction. Various autologous reconstructions have been described, ${ }^{3,5,7}$ and in the instances when free tissue transfer has failed, the source of thrombotic-related failure may be unknown. ${ }^{3,7}$ To the best of our knowledge, we report the first case of deep inferior epigastric perforator (DIEP) flap loss following silicone mastitis reconstruction as a result of free silicone-precipitated thrombosis.

\section{Case Description}

A 37-year-old woman with symptomatic, chronic silicone mastitis from free silicone breast augmentation was evaluated for surgical treatment. Physical examination demonstrated dense and asymmetrically disfigured breast tissue. Ultrasound and mammographic imaging was consistent

\footnotetext{
* Both authors contributed equally to the article.
}

received

December 13, 2016 accepted after revision March 6, 2017 with diffuse granulomatous lesions ( - Fig. 1). She underwent bilateral mastectomy ( - Fig. 2) and autologous reconstruction. Bilateral DIEP flaps were elevated, and the vessels were anastomosed to the right internal mammary (IM) and left IM perforator vessels with successful flap perfusion. The patient was transferred to the surgical intensive care unit for routine hourly flap checks. Fourteen hours postoperatively, Doppler signals became diminished in the left flap and the patient urgently returned to the operating room. Exploration of the flap demonstrated a venous thrombus that was removed with difficulty by Fogarty catheter. The thrombus was sent for pathologic review to determine the etiology of venous thrombosis. Flap salvage failed despite multiple reanastomosis attempts following tissue-plasminogen activator and heparinized saline irrigation. The left breast was reconstructed with a tissue expander. The remainder of her hospital course was uncomplicated. The pathology report confirmed chronic granulomatous lesions in both breasts and silicone particles within the vessel thrombus (-Fig. $\mathbf{3}$ ).

\section{Discussion}

Use of free silicone injections for breast augmentation persists in many parts of the world. Despite the FDA's initial investigation in 1965 concluding free silicone to be safe for soft tissue augmentation, ${ }^{2}$ many reports demonstrate that silicone is not biologically inert. ${ }^{1,5,7,8}$ Over time, silicone migrates into surrounding tissues, causing a chronic granulomatous inflammatory response and pain. ${ }^{1,6}$ To effectively treat these symptoms, most patients with silicone mastitis undergo total mastectomy. Reconstructive options include implant-based and autologous tissue reconstruction. The

\section{Copyright $\odot 2017$ by Thieme Medical Publishers, Inc., 333 Seventh Avenue, New York, NY 10001, USA.

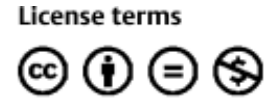
Tel: +1(212) 584-4662.
$10.1055 / \mathrm{s}-0037-1602595$ ISSN 2377-0813. 


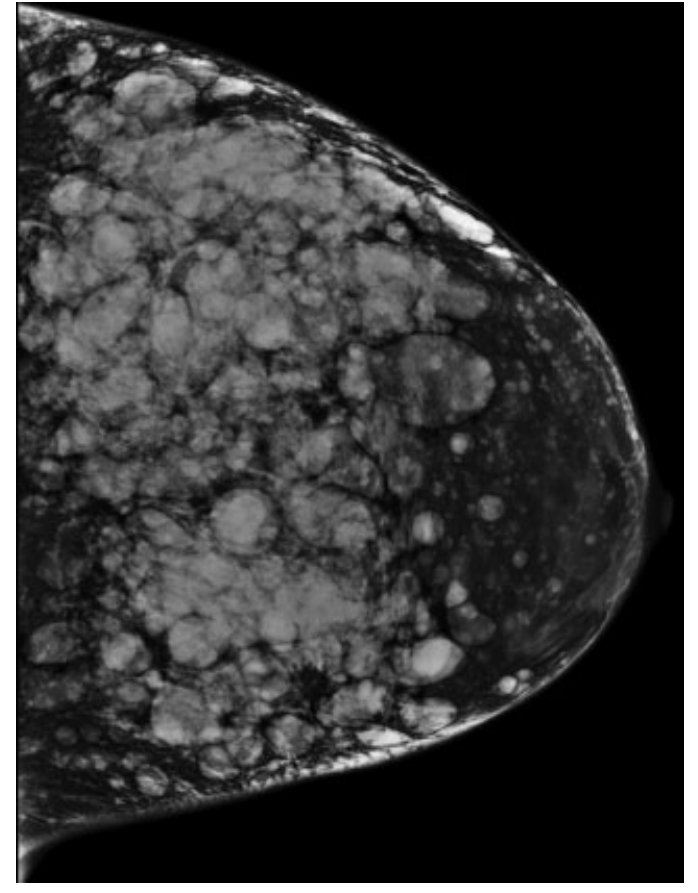

Fig. 1 Left breast craniocaudal mammographic image revealing granulomatous disease following silicone injection.

inflammatory response associated with silicone deposits impairs tissue perfusion and increases the risk of flap infection, necrosis, and tissue contracture. ${ }^{8,9}$ Though silicone gel implant reconstruction is an option, the addition of a foreign body is at risk for implant exposure and infection, as well as capsular contracture, in the setting of silicone-infiltrated mastectomy skin flaps. On the other hand, autologous tissue transfer offers breast reconstruction with new, vascularized soft tissue.

Free tissue breast reconstruction offers greater selection of donor tissue and avoids implant-based morbidity. Flap

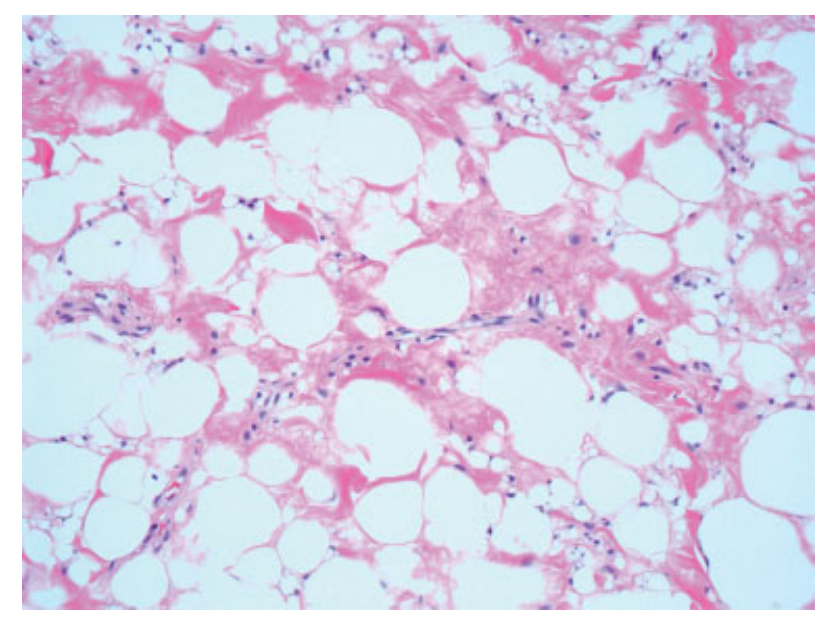

Fig. 2 Histologic specimen of hematoxylin and eosin-stained breast tissue demonstrating diffuse silicone mastitis. Empty spaces were filled with silicone intermixed with adipocytes.

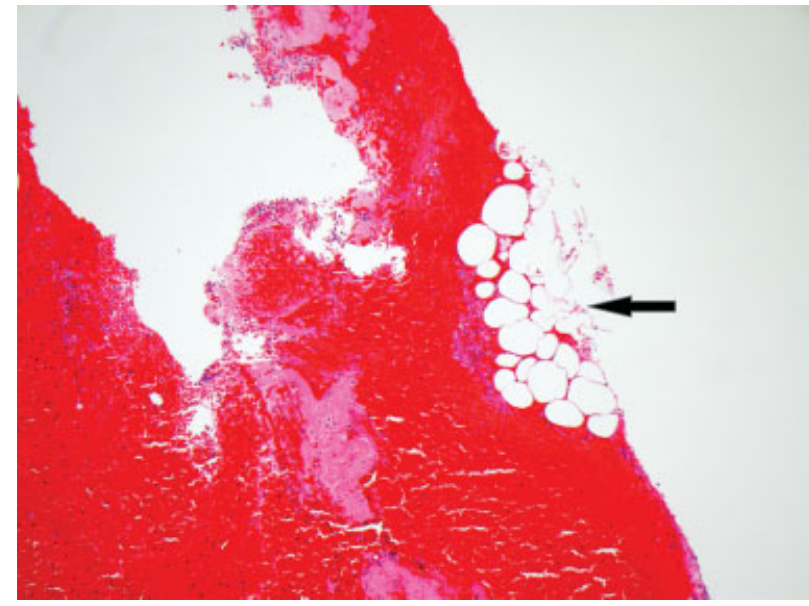

Fig. 3 Silicone precipitated thrombus. Pink indicates layering fibrin consistent with clot. Black arrow indicates conglomerate of silicone within thrombus.

selection is determined by body habitus and breast volume. The transverse rectus abdominis muscle (TRAM), superior gluteal artery perforator (SGAP), and latissimus dorsi (LD) flaps have been used for autologous reconstruction of silicone-related disease, and they have demonstrated comparable results and complications. ${ }^{3,5,7}$

Echo et al demonstrated successful free autologous breast reconstructions for silicone mastitis where most microanastomoses were performed with thoracodorsal (TD) vessels. ${ }^{5}$ However, silicone infiltration of recipient TD vessels in a few patients left them unsuitable for anastomosis and required anastomoses to IM vessels. These authors reported a $0 \%$ flap loss. On the other hand, Mizuno and colleagues reported a concern about chronic axillary inflammation within the "zone of silicone," that is, areas of silicone concentration, where free TRAM flap microanastomoses to TD recipient vessels were performed. Complete necrosis was noted in $7.9 \%$ of flaps and was secondary to venous thrombosis. ${ }^{7}$

In our case, DIEP flap offered an opportunity to use a reliable flap with the lowest risk of hernia development compared with other abdominal-based reconstruction. Use of IM vessels was otherwise expected to be successful as this is the most commonly used recipient site in breast reconstruction. Indeed, the right flap survived. Likewise, IM perforator vessels were selected as Saint-Cyr and colleagues demonstrated that they are equally reliable and do not increase the risk of flap loss, fat necrosis, or mastectomy skin flap necrosis when compared with IM vessels. ${ }^{10}$ However, our left IM perforator vessels, though widely patent, were within the zone of silicone and therefore at higher risk for mechanical introduction of silicone into the vessels compared with deeper IM vessels. Unfortunately, the resulting silicone thrombosis left the flap unsalvageable.

Although pathology review was not included in prior cases series, ${ }^{3,5,7}$ silicone is known to be hydrophobic in nature with an affinity for platelets and fibronectin, which could have precipitated thrombosis. ${ }^{6}$ In our case, the 
anastomotic vessel thrombus was sent for evaluation. The specimen demonstrated a thrombus with acute inflammation and silicone particles in the periphery adjacent to layering fibrin (-Fig. 3). This suggests that free silicone was mechanically introduced into the lumen and incited thrombus formation. To our knowledge, this is the first report of pathology-confirmed silicone particle-precipitated flap failure.

For optimal control of silicone contamination and microanastomosis outcomes, we recommend the following: (1) meticulous resection of all silicone-infiltrated tissue; (2) copious irrigation; (3) isolation of the operative field with moist towels; (4) careful selection and evaluation of recipient vessels; (5) use of new instruments if silicone contamination remains a concern; and (6) vascular anastomosis ideally outside the "zone of silicone."

\section{Conclusion}

This case illustrates the first reported incident of free flap failure due to pathology-confirmed silicone particle-precipitated thrombosis. Mastectomy with free flap reconstruction represents a viable option for silicone mastitis, but a high degree of vigilance should be maintained to clear the surgical bed of remaining silicone to minimize risk of flap failure.

\section{References}

1 Peters W, Fornasier V. Complications from injectable materials used for breast augmentation. Can J Plast Surg 2009;17(03):89-96

2 Coleman SR. Injectable silicone returns to the United States. Aesthet Surg J 2001;21(06):576-578

3 Feng L-J, Mauceri K, Berger BE. Autogenous tissue breast reconstruction in the silicone-intolerant patient. Cancer 1994; 74(1, Suppl):440-449

4 Boo-Chai K. The complications of augmentation mammaplasty by silicone injection. Br J Plast Surg 1969;22(03):281-285

5 Echo A, Otake LR, Mehrara BJ, et al. Surgical management of silicone mastitis: case series and review of the literature. Aesthetic Plast Surg 2013;37(04):738-745

6 Yoshida SH, Swan S, Teuber SS, Gershwin ME. Silicone breast implants: immunotoxic and epidemiologic issues. Life Sci 1995; 56(16):1299-1310

7 Mizuno H, Hyakusoku H, Fujimoto M, Kawahara S, Aoki R. Simultaneous bilateral breast reconstruction with autologous tissue transfer after the removal of injectable artificial materials: a 12-year experience. Plast Reconstr Surg 2005;116(02):450-458

8 Lai YL, Weng CJ, Noordhoff MS. Breast reconstruction with TRAM flap after subcutaneous mastectomy for injected material (siliconoma). Br J Plast Surg 2001;54(04):331-334

9 Chiu W-K, Lee TP, Chen SY, Li CC, Wang CH, Chen S-G. Bilateral breast reconstruction with a pedicled transverse rectus abdominis myocutaneous flap after subcutaneous mastectomy for symptomatic injected breasts. J Plast Surg Hand Surg 2012;46(3-4):242-247

10 Saint-Cyr M, Chang DW, Robb GL, Chevray PM. Internal mammary perforator recipient vessels for breast reconstruction using free TRAM, DIEP, and SIEA flaps. Plast Reconstr Surg 2007;120(07): 1769-1773 